University of Wollongong

Research Online

Faculty of Informatics - Papers (Archive)

Faculty of Engineering and Information

Sciences

July 2005

\title{
MMSE-optimal approximation of continuous-phase modulated signal as superposition of linearly modulated pulses
}

Xiaojing Huang

University of Wollongong, huang@uow.edu.au

Y. Li

University of Wollongong

Follow this and additional works at: https://ro.uow.edu.au/infopapers

Part of the Physical Sciences and Mathematics Commons

\section{Recommended Citation}

Huang, Xiaojing and Li, Y.: MMSE-optimal approximation of continuous-phase modulated signal as superposition of linearly modulated pulses 2005.

https://ro.uow.edu.au/infopapers/165

Research Online is the open access institutional repository for the University of Wollongong. For further information contact the UOW Library: research-pubs@uow.edu.au 


\title{
MMSE-optimal approximation of continuous-phase modulated signal as superposition of linearly modulated pulses
}

\author{
Abstract \\ The optimal linear modulation approximation of any M-ary continuous-phase modulated (CPM) signal \\ under the minimum mean-square error (MMSE) criterion is presented in this paper. With the introduction \\ of the MMSE signal component, an M-ary CPM signal is exactly represented as the superposition of a \\ finite number of MMSE incremental pulses, resulting in the novel switched linear modulation CPM signal \\ models. Then, the MMSE incremental pulse is further decomposed into a finite number of MMSE pulse- \\ amplitude modulated (PAM) pulses, so that an M-ary CPM signal is alternatively expressed as the \\ superposition of a finite number of MMSE PAM components, similar to the Laurent representation. \\ Advantageously, these MMSE PAM components are mutually independent for any modulation index. The \\ optimal CPM signal approximation using lower order MMSE incremental pulses, or alternatively, using a \\ small number of MMSE PAM pulses, is also made possible, since the approximation error is minimized in \\ the MMSE sense. Finally, examples of the MMSE-optimal CPM signal approximation and its comparison \\ with the Laurent approximation approach are given using raised-cosine frequency-pulse CPM schemes.

\section{Keywords} \\ continuous phase modulation, least squares approximations, pulse amplitude modulation, signal \\ processing

\section{Disciplines} \\ Physical Sciences and Mathematics

\section{Publication Details} \\ This paper originally appeared as: Huang, $X$ and Li, Y, MSE-optimal approximation of continuous-phase \\ modulated signal as superposition of linearly modulated pulses, IEEE Transactions on Communications, \\ July 2005, 53(7), 1166-1177. Copyright IEEE 2005.
}




\title{
MMSE-Optimal Approximation of Continuous-Phase Modulated Signal as Superposition of Linearly Modulated Pulses
}

\author{
Xiaojing Huang, Member, IEEE, and Yunxin Li, Senior Member, IEEE
}

\begin{abstract}
The optimal linear modulation approximation of any $M$-ary continuous-phase modulated (CPM) signal under the minimum mean-square error (MMSE) criterion is presented in this paper. With the introduction of the MMSE signal component, an $M$-ary CPM signal is exactly represented as the superposition of a finite number of MMSE incremental pulses, resulting in the novel switched linear modulation CPM signal models. Then, the MMSE incremental pulse is further decomposed into a finite number of MMSE pulse-amplitude modulated (PAM) pulses, so that an $M$-ary CPM signal is alternatively expressed as the superposition of a finite number of MMSE PAM components, similar to the Laurent representation. Advantageously, these MMSE PAM components are mutually independent for any modulation index. The optimal CPM signal approximation using lower order MMSE incremental pulses, or alternatively, using a small number of MMSE PAM pulses, is also made possible, since the approximation error is minimized in the MMSE sense. Finally, examples of the MMSE-optimal CPM signal approximation and its comparison with the Laurent approximation approach are given using raised-cosine frequency-pulse CPM schemes.
\end{abstract}

Index Terms-Continuous-phase modulation (CPM), Laurent representation, minimum mean-square error (MMSE).

\section{INTRODUCTION}

$\mathbf{C}$ ONTINUOUS-PHASE modulation (CPM) [1]-[4] is a nonlinear modulation scheme, although the phase responds linearly to the input data. CPM has the advantage of excellent power and bandwidth efficiency with constant signal envelope. Its disadvantage is its nonlinear nature, which results in complexity in receiver implementation and difficulty in signal analysis. For these reasons, the CPM scheme has been mostly used for binary signaling with special modulation indexes in many power- and bandwidth-efficient communication systems. To combat the inherent nonlinearity, Laurent discovered that a binary CPM signal with any noninteger modulation index can be exactly decomposed as a sum of a finite number of pulse-amplitude modulated (PAM) signal components [5]. Mengali and Morelli extended this binary PAM decomposition to multilevel cases by expressing a noninteger modulation

Paper approved by G. M. Vitetta, the Editor for Equalization and Fading Channels of the IEEE Communications Society. Manuscript received March 11, 2003; revised May 30, 2003; June 29, 2004; July 28, 2004; and February 10,2005 .

$\mathrm{X}$. Huang is with the School of Electrical, Computer, and Telecommunications Engineering, Faculty of Informatics, University of Wollongong, Wollongong, NSW 2522, Australia (e-mail: huang@uow.edu.au).

Y. Li is with the Twincall Education Center, Twincall Pty Ltd., Ryde, NSW 2112, Australia (e-mail: jeff@twincall.com).

Digital Object Identifier 10.1109/TCOMM.2005.851625 index $M$-ary CPM signal as the product of several constitutional binary CPM signals [6]. A complementary solution for decomposing the integer modulation index CPM signal was also proposed recently [7]. Thus, the theory of CPM signal PAM decomposition under the banner of Laurent representation seemed to be complete.

However, the Laurent representation has two major drawbacks. First, the PAM components of a CPM signal are generally not mutually independent, except when the modulation index is a multiple of 0.5 . As a consequence, the autocorrelation function of a CPM signal is not, in general, a sum of all PAM pulses' autocorrelation functions. Cross-correlations between different PAM components exist, which still causes difficulty in CPM signal analysis. Second, when a small number of PAM pulses are used to approximate a CPM signal, the approximation error is not minimized in the minimum mean-square error (MMSE) sense, except for some special modulation indexes. Other drawbacks include the inefficiency in practical application, due to the large number of pseudosymbols in which the data symbols are hidden, and the complicated decomposition procedure, especially for multilevel CPM signals.

In this paper, a new approach, directly applicable to any $M$-ary CPM signal, is taken to represent the CPM signal as the superposition of linearly modulated pulses. By this approach, novel switched linear modulation models are derived for the CPM signal with either an integer or noninteger modulation index. Then, the linearly modulated pulses in the models are further decomposed into PAM pulses, similar to Laurent representation. However, the PAM components are now always mutually independent. When fewer linearly modulated pulses are used to approximate the CPM signal, with a simultaneous reduction in signal memory, the approximation error is always minimized in the MMSE sense. Other advantages include the explicit closed-form expressions for all functions and coefficients in the representation. Thus, the drawbacks associated with the Laurent representation as mentioned above are all solved.

As has been known to us, many existing simplified CPM models, such as Laurent's PAM decomposition and Rimoldi's continuous-phase encoder (CPE) plus memoryless modulator (MM) decomposition [13], have found wide application in CPM signal analysis, receiver complexity reduction, signal synchronization, and parameter estimation [8]-[13]. The proposed switched linear modulation models can be also exploited to construct simple CPM receivers, reducing both the number of matched filters and trellis states at the same time [14]. 
It is worth noting that in terms of matched-filter reduction for the maximum-likelihood-type CPM receivers, Huber and Liu took a signal space dimension reduction approach [15]. Moqvist and Aulin showed that the optimal lower dimensional approximation was given by the principal components method under the criterion of minimum residual error [16]. However, this approach does not reduce the memory of the approximated CPM signal, so the reduction in trellis states has to be treated separately.

The rest of this paper is organized as follows. Analogous to the Laurent signal component defined in Appendix A, the notion of an MMSE signal component for an $M$-ary CPM signal is first introduced in Section II. Then, the switched linear modulation models are derived in Section III by decomposing the CPM signal as a superposition of the MMSE incremental pulses. In Section IV, the MMSE incremental pulses are further decomposed into a finite number of MMSE PAM pulses associated with independent pseudosymbols. In Section V, the autocorrelation function of the CPM signal approximated by lower order MMSE incremental pulses is formulated, and the approximation error is proven to be minimized. In Section VI, examples of the MMSE-optimal CPM signal approximation and the comparison with the Laurent approximation approach are given using the raised-cosine frequency pulse (LRC) CPM scheme. Finally, conclusions are drawn in Section VII. A reformulated Laurent representation is also provided in Appendix B.

\section{MMSE SignAl COMPONENT OF CPM SIGNAL}

The equivalent lowpass envelope of an $M$-ary CPM signal [1]-[4] with unity signal power can be expressed as

$$
S(t)=e^{j \sum_{n=-\infty}^{\infty} a_{n} \varphi(t-n T)}
$$

where the data symbol $a_{n}$ with symbol interval $T$ belongs to the $M$-ary alphabet $[ \pm 1, \pm 3, \ldots, \pm(M-1)]$, and the phase shift function $\varphi(t)$ is assumed to be zero for a negative value of time and $h \pi$ ( $h$ denotes the modulation index) for time greater than $L$ symbol intervals, i.e.,

$$
\varphi(t)=\left\{\begin{array}{ll}
0, & \text { for } t \leq 0 \\
h \pi, & \text { for } t \geq L T
\end{array}, \quad L \geq 1 .\right.
$$

For $0 \leq t \leq L T, \varphi(t)$ can be any monotonic function. According to the Laurent representation [5]-[7], $S(t)$ for noninteger $h$ can be decomposed into a sum of a finite number of PAM components. The coefficient, denoted as $a_{k, n}$, associated with the $k$ th Laurent PAM pulse at time $t=n T$ is referred to as the pseudosymbol [6]. Especially, the pseudosymbol associated with the first Laurent PAM pulse $(k=0)$ can be expressed as

$$
\begin{aligned}
a_{0, n} & =e^{j h \pi \sum_{i=-\infty}^{n} a_{i}}=a_{0, n-1} e^{j h \pi a_{n}} \\
& =\cdots=a_{0, n-l} e^{j h \pi \sum_{i=n-l+1}^{n} a_{i}}, \quad l>0
\end{aligned}
$$

which represents the CPM signal's accumulative phase rotation contributed by all previously transmitted data symbols up to time $t=n T$. Since all the possible phases of $a_{0, n}$ constitute the phase states of the CPM signal, we simply refer to it as the phase state symbol.

Now let us consider the contribution of $l$ consecutively transmitted data symbols $a_{N-l+1}, a_{N-l+2}, \ldots, a_{N}$, starting from time $t=(N-l+1) T$ where $N$ stands for a specific time index, to the transmitted CPM signal waveform. We refer to this contribution as a kind of signal component associated with the $l$ data symbols. The expression of this signal component must meet the following requirements. First, this signal component is a function of the $l$ data symbols. Second, due to the CPM's phase continuity, this signal component should contain a factor $a_{0, N-l}$ to account for the influence (phase rotation) of all previously transmitted data symbols up to time $t=(N-l) T$. In Appendix A, we have shown that the Laurent signal component $S\left(t, a_{N-l+1}, a_{N-l+2}, \ldots, a_{N}\right)$ is exactly the product of the Laurent complex pulse $S_{a_{N-l+1}, a_{N-l+2}, \ldots, a_{N}}(t)$, which is a function of $l$ data symbols, with $a_{0, N-l}$ (for noninteger $h$ ) or $e^{j h \pi N}$ (for integer $h$ ), see (A4) and (A5).

Thus, analogous to the Laurent signal component, we define the MMSE signal component $\bar{S}\left(t, a_{N-l+1}, a_{N-l+2}, \ldots, a_{N}\right)$ in the form of

$$
\begin{aligned}
& \bar{S}\left(t, a_{N-l+1}, a_{N-l+2}, \ldots, a_{N}\right) \\
& \quad=a_{0, N-l} \bar{S}_{a_{N-l+1}, a_{N-l+2}, \ldots, a_{N}}(t-N T), \text { for noninteger } h
\end{aligned}
$$

$$
\begin{aligned}
& \bar{S}\left(t, a_{N-l+1}, a_{N-l+2}, \ldots, a_{N}\right) \\
& \quad=e^{j h \pi N} \bar{S}_{a_{N-l+1}, a_{N-l+2}, \ldots, a_{N}}(t-N T), \text { for integer } h
\end{aligned}
$$

but determine the function $\bar{S}_{a_{N-l+1}, a_{N-l+2}, \ldots, a_{N}}(t)$ by the MMSE criterion. Under this criterion, we first express the mean-square error (MSE) between the $M$-ary CPM signal $S(t)$ and its MMSE signal component

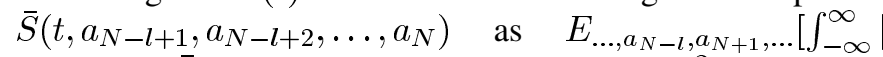
$\left.S(t)-\left.\bar{S}\left(t, a_{N-l+1}, a_{N-l+2}, \ldots, a_{N}\right) \quad\right|^{2} d t\right]$, where

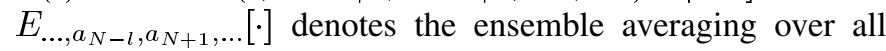
symbols other than $a_{N-l+1}, a_{N-l+2}, \ldots, a_{N}$. Then, letting

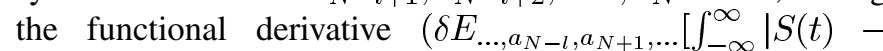
$\left.\left.\left.\bar{S}\left(t, a_{N-l+1}, a_{N-l+2}, \ldots, a_{N}\right)\right|^{2} d t\right]\right) /\left(\delta \bar{S}_{a_{N-l+1}, a_{N-l+2}, \ldots, a_{N}}\right.$ $(t))=0$ to minimize the MSE, we find

$$
\begin{aligned}
& \bar{S}_{a_{N-l+1}, a_{N-l+2}, \ldots, a_{N}}(t) \\
& \quad=E_{\ldots, a_{N-l}, a_{N+1}, \ldots}\left[S(t+N T) a_{0, N-l}^{*}\right] \\
& \quad=\bar{w}_{l}(t) \cdot e^{j \sum_{i=-l+1}^{0} a_{N+i} \varphi(t-i T)}, \quad \text { for noninteger } h
\end{aligned}
$$

where

$$
\bar{w}_{l}(t)=\prod_{i=-\infty}^{-l} \frac{\sin M[h \pi-\varphi(t-i T)]}{M \sin [h \pi-\varphi(t-i T)]} \prod_{i=1}^{\infty} \frac{\sin M \varphi(t-i T)}{M \sin \varphi(t-i T)}
$$

We see that $\bar{S}_{a_{N-l+1}, a_{N-l+2}, \ldots, a_{N}}(t)$ is also a windowed complex exponential similar to the Laurent complex pulse derived in Appendix A, and is therefore referred to as the MMSE complex pulse. Accordingly, $\bar{w}_{l}(t)$ is called the MMSE window function. Applying the same MMSE criterion for an $M$-ary CPM signal with integer $h$, the MMSE complex pulse and the MMSE window function have the same expressions as (6) and (7), respectively, after ignoring the sign ambiguity for odd $h$ due to an undetermined number of factors $\cos (h \pi)(=-1$ for odd $h)$ involved in the derivation. 
Derived from (7) and (2), $\bar{w}_{l}(t)$ demonstrates some interesting properties, such as

$$
\begin{aligned}
& \bar{w}_{l}(t) \frac{\sin M[h \pi-\varphi(t+l T-T)]}{M \sin [h \pi-\varphi(t+l T-T)]}=\bar{w}_{l-1}(t) \\
& \bar{w}_{l}(t) \frac{\sin M \varphi(t)}{M \sin \varphi(t)}=\bar{w}_{l-1}(t+T) \\
& \bar{w}_{l}(t)=\bar{w}_{l-1}(t), \quad \text { for } t \geq(L-l+1) T .
\end{aligned}
$$

$\bar{w}_{l}(t)$ generally has an infinite duration (except for $h=q / M$, where $q$ is any integer), but will attenuate to zero for noninteger $h$ as $t \rightarrow \pm \infty$. For integer $h, \bar{w}_{l}(t)$ oscillates as $t \rightarrow \pm \infty$. If $l=0, \bar{w}_{0}(t)$ with integer $h$ becomes a periodic function, so that it is alternatively expressed as

$$
\bar{w}_{0}(t)=\sum_{n=-\infty}^{\infty} e^{j h \pi n} h_{0}(t-n T), \quad \text { for integer } h
$$

where

$$
h_{0}(t)= \begin{cases}\prod_{i=-L+1}^{0} \frac{\sin M \varphi(t-i T)}{M \sin \varphi(t-i T)}, & 0 \leq t \leq T \\ 0, & \text { elsewhere }\end{cases}
$$

is one ( $h$ even) or half ( $h$ odd) period of $\bar{w}_{0}(t)$.

\section{CPM SigNAL REPRESENTATION BY MMSE INCREMENTAL PULSES}

To decompose an $M$-ary CPM signal into additive linearly modulated pulses, let us first analyze the difference between the MMSE signal component associated with $l$ data symbols $a_{N-l+1}, a_{N-l+2}, \ldots, a_{N}$ and the one associated with $l-1$ data symbols $a_{N-l+1}, a_{N-l+2}, \ldots, a_{N-1}$, which indicates an increment to the waveform of the MMSE signal component when the data symbol $a_{N}$ is transmitted after $a_{N-l+1}, a_{N-l+2}, \ldots, a_{N-1}$. Assuming a noninteger $h$ and using the property of $\bar{w}_{l}(t)$ given in (9), this difference is found to be

$$
\begin{aligned}
\bar{S}\left(t, a_{N-l+1}, a_{N-l+2}, \ldots, a_{N}\right) \\
\quad-\bar{S}\left(t, a_{N-l+1}, a_{N-l+2}, \ldots, a_{N-1}\right) \\
=a_{0, N-l} \bar{w}_{l}(t-N T) \\
\quad \cdot e^{j \sum_{i=-l+1}^{0} a_{N+i} \varphi(t-N T-i T)} \\
\quad-a_{0, N-l} \bar{w}_{l-1}(t-N T+T) \\
\quad \cdot e^{j \sum_{i=-l+1}^{-1} a_{N+i} \varphi(t-N T-i T)} \\
=a_{0, N-1} h_{a_{N-l+1}, a_{N-l+2}, \ldots, a_{N}}(t-N T)
\end{aligned}
$$

where

$$
\begin{aligned}
h_{a_{N-l+1}, a_{N-l+2}, \ldots, a_{N}}(t) \\
=\bar{w}_{l}(t) \cdot e^{-j \sum_{i=-l+1}^{-1} a_{N+i}[h \pi-\varphi(t-i T)]} \\
\quad \cdot\left(e^{j a_{N} \varphi(t)}-\frac{\sin M \varphi(t)}{M \sin \varphi(t)}\right)
\end{aligned}
$$

is a causal function (i.e., $h_{a_{N-l+1}, a_{N-l+2}, \ldots, a_{N}}(t)=0$ for $t<0$ ) and is referred to as the MMSE incremental pulse of order $l$. Note that for any $l>L$, the $l$ th-order MMSE incremental pulse $h_{a_{N-l+1}, a_{N-l+2}, \ldots, a_{N}}(t)$ will be the same as the $L$ th-order one $h_{a_{N-L+1}, a_{N-L+2}, \ldots, a_{N}}(t)$, since $\bar{w}_{l}(t)=\bar{w}_{L}(t)$ and $h \pi-\varphi(t+$ $l T)=0$ for $t \geq 0$ and $l>L$, according to (10) and (2).
Then we rewrite the MMSE signal component in terms of the MMSE incremental pulses of orders from 1 to $L$ as

$$
\begin{aligned}
\bar{S}(t, & \left.a_{N-l+1}, a_{N-l+2}, \ldots, a_{N}\right) \\
= & a_{0, N-l} \bar{w}_{0}(t-(N-l) T) \\
& +\left[\bar{S}\left(t, a_{N-l+1}\right)-a_{0, N-l} \bar{w}_{0}(t-(N-l) T)\right] \\
& +\left[\bar{S}\left(t, a_{N-l+1}, a_{N-l+2}\right)-\bar{S}\left(t, a_{N-l+1}\right)\right]+\cdots \\
& +\left[\bar{S}\left(t, a_{N-l+1}, a_{N-l+2}, \ldots, a_{N-l+L}\right)\right. \\
& \left.-\bar{S}\left(t, a_{N-l+1}, a_{N-l+2}, \ldots, a_{N-l+L-1}\right)\right] \\
& +\left[\bar{S}\left(t, a_{N-l+1}, a_{N-l+2}, \ldots, a_{N-l+L+1}\right)\right. \\
& \left.-\bar{S}\left(t, a_{N-l+1}, a_{N-l+2}, \ldots, a_{N-l+L}\right)\right]+\ldots \\
& +\left[\bar{S}\left(t, a_{N-l+1}, a_{N-l+2}, \ldots, a_{N}\right)\right. \\
& \left.-\bar{S}\left(t, a_{N-l+1}, a_{N-l+2}, \ldots, a_{N-1}\right)\right] \\
= & a_{0, N-l} \bar{w}_{0}(t-(N-l) T) \\
& +a_{0, N-l} h_{a_{N-l+1}}(t-(N-l+1) T) \\
& +a_{0, N-l+1} h_{a_{N-l+1}, a_{N-l+2}}(t-(N-l+2) T)+\cdots \\
& +a_{0, N-l+L-1} h_{a_{N-l+1}, a_{N-l+2}, \ldots, a_{N-l+L}} \\
& (t-(N-l+L) T) \\
& +a_{0, N-l+L} h_{a_{N-l+2}, a_{N-l+3}, \ldots, a_{N-l+L+1}} \\
& (t-(N-l+L+1) T)+\cdots \\
& +a_{0, N-1} h_{a_{N-L+1}, a_{N-L+2}, \ldots, a_{N}}(t-N T) .
\end{aligned}
$$

Letting $l \rightarrow \infty$, the MMSE signal component will be expressed as the convolution of only the $L$ th-order MMSE incremental pulse with the phase state symbol, since the first $L$ terms on the right-hand side (RHS) of (14) will decay to zero because of the attenuated window functions, that is

$$
\begin{aligned}
\bar{S}\left(t, a_{-\infty}, \ldots, a_{N-2}, a_{N-1}, a_{N}\right) \\
=\sum_{n=-\infty}^{N} a_{0, n-1} h_{a_{n-L+1}, a_{n-L+2}, \ldots, a_{n}}(t-n T) .
\end{aligned}
$$

Finally, letting $N \rightarrow \infty$, the MMSE signal component becomes $\bar{S}\left(t, a_{-\infty}, \ldots, a_{n-2}, a_{n-1}, a_{n}, \ldots, a_{\infty}\right)$, which is the $M$-ary CPM signal itself (assuming an initial value of the phase state symbol $\left.a_{0,-\infty}=1\right)$, i.e.,

$$
\begin{aligned}
S(t) & =\bar{S}\left(t, a_{-\infty}, \ldots, a_{n-2}, a_{n-1}, a_{n}, \ldots, a_{\infty}\right) \\
& =\sum_{n=-\infty}^{\infty} a_{0, n-1} h_{a_{n-L+1}, a_{n-L+2}, \ldots, a_{n}}(t-n T) .
\end{aligned}
$$

In this way, the $M$-ary CPM signal is decomposed exactly into a sum of the $L$ th-order MMSE incremental pulses $h_{a_{n-L+1}, a_{n-L+2}, \ldots, a_{n}}(t-n T)$ modulated by the phase state symbols $a_{0, n-1}$.

Following a similar procedure as described above and carefully dealing with the sign ambiguity, we can also decompose the $M$-ary CPM signal with integer $h$ into a sum of the $L$ th-order MMSE incremental pulses. However, since $\bar{w}_{0}(t)$ becomes a periodic function with integer $h$ [see (11)], it will not decay to zero, but will appear in the final decomposition expression. Thus

$$
\begin{aligned}
S(t)= & \sum_{n=-\infty}^{\infty} e^{j h \pi n} h_{0}(t-n T) \\
& +\sum_{\substack{n=-\infty \\
\text { for integer } h .}}^{\infty} e^{j h \pi n} h_{a_{n-L+1}, a_{n-L+2}, \ldots, a_{n}}(t-n T)
\end{aligned}
$$




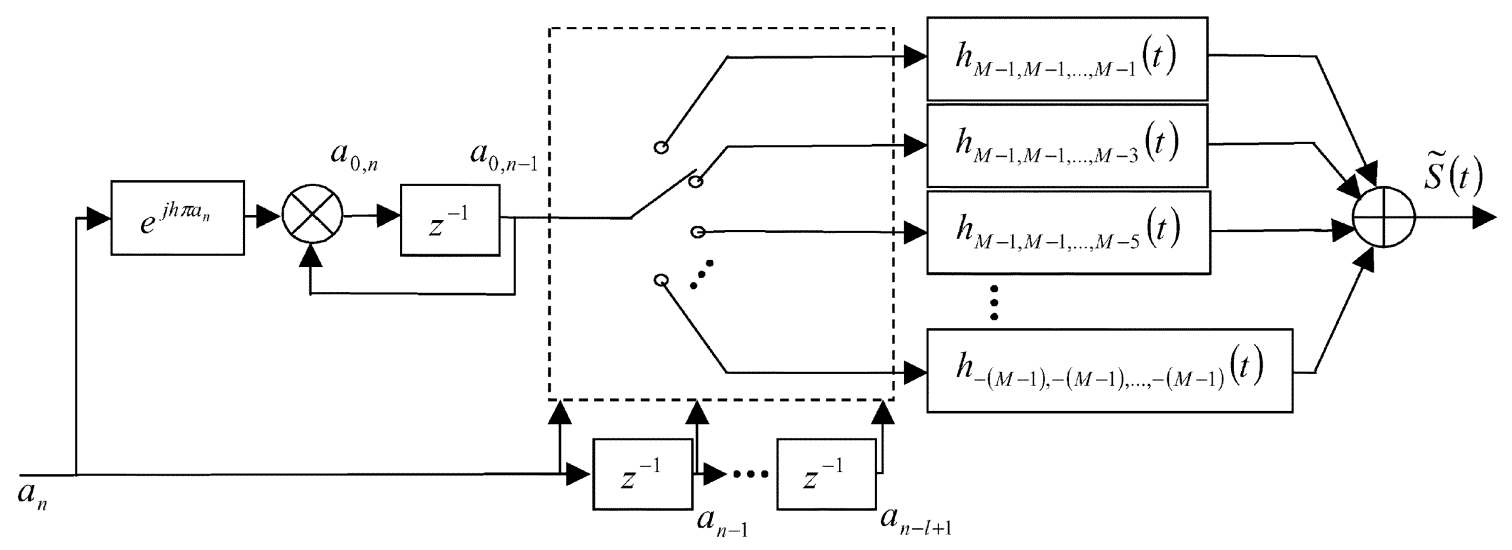

(a)

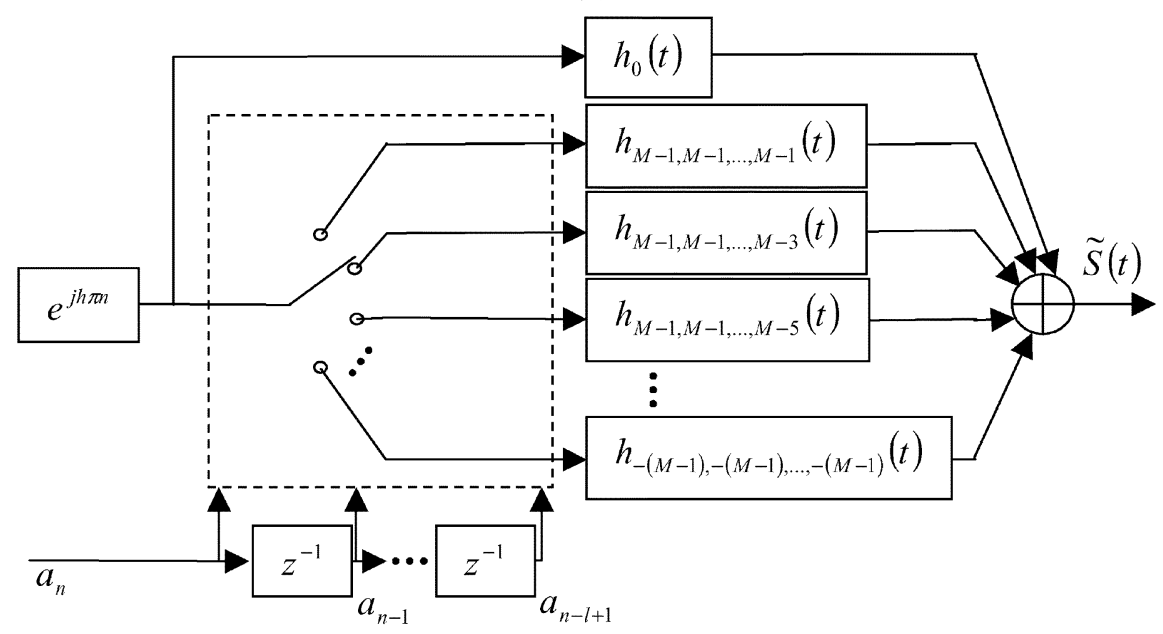

(b)

Fig. 1. Switched linear modulation models. (a) For noninteger modulation index CPM signal. (b) For integer modulation index CPM signal.

Equations (16) and (17) represent the exact modeling of an $M$-ary CPM signal using the $L$ th-order MMSE incremental pulses. To have more efficient signal modeling, we may wish to use the MMSE incremental pulses of lower order $l<L$ to approximate the $M$-ary CPM signal, i.e.,

$$
\begin{aligned}
S(t) \approx \tilde{S}(t)= & \sum_{n=-\infty}^{\infty} a_{0, n-1} h_{a_{n-l+1}, a_{n-l+2}, \ldots, a_{n}}(t-n T), \\
\text { for noninteger } h & \\
S(t) \approx \tilde{S}(t)= & \sum_{n=-\infty}^{\infty} e^{j h \pi n} h_{0}(t-n T) \\
& +\sum_{n=-\infty}^{\infty} e^{j h \pi n} h_{a_{n-l+1}, a_{n-l+2}, \ldots, a_{n}}(t-n T),
\end{aligned}
$$

for integer $h$.

Equations (18) and (19) suggest new signal models for the CPM signal representation and approximation. We call these models switched linear modulation models, which are illustrated in Fig. 1. For a noninteger modulation index CPM signal, the switched linear modulation model comprises a switch and a bank of $M^{l}$ filters with the MMSE incremental pulses $h_{a_{n-l+1}, a_{n-l+2}, \ldots, a_{n}}(t)$ as their respective impulse responses. The input symbol $a_{n}$ and the previously transmitted symbols $a_{n-l+1}, a_{n-l+2}, \ldots, a_{n-1}$ stored in an $l-1$ stage shift register (representing the memory) are applied to the switch to select the modulation filter, and the phase state symbol $a_{0, n-1}$ is fed into the selected filter. All the filters' outputs are finally added together to give the approximated CPM signal. For an integer modulation index CPM signal, a filter with impulse response $h_{0}(t)$ is always connected to generate the periodic component, in addition to the switch and the bank of $M^{l}$ filters. The data-independent phase state symbol $e^{j h \pi n}$ is fed into the filter $h_{0}(t)$ and the linear modulation filter, selected according to data symbols $a_{n-l+1}, a_{n-l+2}, \ldots, a_{n-1}$, and $a_{n}$. With the above switched linear modulation models, CPM is now described as a linear time-varying system. The nonlinearity inherent within the phase modulation is embodied in the phase state symbol $a_{0, n-1}$.

In all $M^{l}$ complex-valued MMSE incremental pulses of order $l$, there are only $M^{l-1}(M-1)$ independent real-valued pulses, due to the properties of $h_{a_{n-l+1}, a_{n-l+2}, \ldots, a_{n}}(t)$ derived from (13), i.e., $h_{a_{n-l+1}, a_{n-l+2}, \ldots, a_{n}}(t)=h_{-a_{n-l+1},-a_{n-l+2}, \ldots,-a_{n}}^{*}(t)$ (symmetry property) and $E_{a_{n}}\left[h_{a_{n-l+1}, a_{n-l+2}, \ldots, a_{n}}(t)\right]=0$, where $E_{a_{n}}[\cdot]$ denotes the ensemble averaging over $a_{n}$.

\section{CPM SignAl REPRESENTATION By MMSE PAM PULSES}

The $l$ th-order MMSE incremental pulse $h_{a_{n-l+1}, a_{n-l+2}, \ldots, a_{n}}(t)$ can be further decomposed into additive pulses, so that an $M$-ary CPM signal can be 
alternatively expressed as a sum of a finite number of PAM components, similar to the Laurent representation [5]-[7], but with a different set of PAM pulses and their associated pseudosymbols.

To perform this PAM decomposition, we rewrite (13) as

$$
\begin{aligned}
& h_{a_{n-l+1}, a_{n-l+2}, \ldots, a_{n}}(t) \\
& =\bar{w}_{l}(t)\left[e^{j a_{n} \varphi(t)-j \sum_{i=1}^{l-1} a_{n-i}[h \pi-\varphi(t+i T)]}\right. \\
& \left.\quad-\frac{\sin M \varphi(t)}{M \sin \varphi(t)} e^{-j \sum_{i=1}^{l-1} a_{n-i}[h \pi-\varphi(t+i T)]}\right] .
\end{aligned}
$$

The first complex exponential in the brackets on the RHS of (20) can be expressed as a product of $l$ terms, i.e., $e^{j a_{n} \varphi(t)-j \sum_{i=1}^{l-1} a_{n-i}[h \pi-\varphi(t+i T)]}=e^{j a_{n} \varphi(t)} \prod_{i=1}^{l-1}$ $e^{-j a_{n-i}[h \pi-\varphi(t+i T)]}$. Assuming that $M$ is an integer power of 2, i.e., $M=2^{P}$ where $P$ is any positive integer, the $M$-ary data symbol $a_{n-i}, i=0,1, \ldots, l-1$, can be represented in radix-2 form as

$$
a_{n-i}=\sum_{\rho=0}^{P-1} a_{n-i}^{(\rho)} 2^{\rho}
$$

where $a_{n-i}^{(\rho)} \in\{+1,-1\}$ are binary bits associated with $a_{n-i}$. Thus, a term in the form of $e^{j a_{n} \varphi(t)}$ can be further expressed as a product of $P$ terms, i.e., $e^{j a_{n} \varphi(t)}=$ $e^{j \sum_{\rho=0}^{P-1} a_{n}^{(\rho)} 2^{\rho} \varphi(t)}=\prod_{\rho=0}^{P-1} e^{j a_{n}^{(\rho)} 2^{\rho} \varphi(t)}$, and then expressed as $e^{j a_{n} \varphi(t)}=\prod_{\rho=0}^{P-1}\left[\cos 2^{\rho} \varphi(t)+j a_{n}^{(\rho)} \sin 2^{\rho} \varphi(t)\right]$, since $e^{j a_{n}^{(\rho)} 2^{\rho} \varphi(t)}=\cos 2^{\rho} \varphi(t)+j a_{n}^{(\rho)} \sin 2^{\rho} \varphi(t)$. Applying the above procedure to other terms in the form of $e^{-j a_{n-i}[h \pi-\varphi(t+i T)]}$, we have

$$
\begin{aligned}
e^{j a_{n} \varphi(t)-j \sum_{i=1}^{l-1} a_{n-i}[h \pi-\varphi(t+i T)]} & \\
= & \prod_{\rho=0}^{P-1}\left[\cos 2^{\rho} \varphi(t)+j a_{n}^{(\rho)} \sin 2^{\rho} \varphi(t)\right] \\
& \cdot \prod_{i=1}^{l-1} \prod_{\rho=0}^{P-1}\left\{\cos 2^{\rho}[h \pi-\varphi(t+i T)]\right. \\
& \left.-j a_{n-i}^{(\rho)} \sin 2^{\rho}[h \pi-\varphi(t+i T)]\right\} .
\end{aligned}
$$

Furthermore, a product of $P$ terms in the form of $\prod_{\rho=0}^{P-1}\left[\cos 2^{\rho} \varphi(t)+j a_{n}^{(\rho)} \sin 2^{\rho} \varphi(t)\right]$ can be expanded into a sum of $2^{P}(=M)$ additive terms. Expanding other products of $P$ terms in the form of $\prod_{\rho=0}^{P-1}\left\{\cos 2^{\rho}[h \pi-\varphi(t+i T)]-j a_{n-i}^{(\rho)}\right.$ $\left.\sin 2^{\rho}[h \pi-\varphi(t+i T)]\right\}$ in (22) into respective sums of $M$ additive terms, (22) becomes a product of $l$ sums, each of which has $M$ additive terms, and can be further expanded into a sum of $M^{l}$ additive terms.

To analytically describe this expansion, we use an integer number $k=0,1, \ldots, M^{l}-1$ as the index for the $M^{l}$ additive terms, and represent it in the radix- $M$ form as

$$
k=\sum_{i=0}^{l-1} \beta_{k, i} M^{i}
$$

where $\beta_{k, i}=0,1, \ldots, M-1$, and is further represented in the radix-2 form as

$$
\beta_{k, i}=\sum_{\rho=0}^{P-1} \gamma_{\beta_{k, i}, \rho} 2^{\rho}
$$

where $\gamma_{\beta_{k, i}, \rho}=0,1$. The sum of the $M$ additive terms expanded from $\prod_{\rho=0}^{P-1}\left[\cos 2^{\rho} \varphi(t)+j a_{n}^{(\rho)} \sin 2^{\rho} \varphi(t)\right]$ is then expressed as $\sum_{\beta_{k, 0}=0}^{M-1}\left\{\prod_{\rho=0}^{P-1}\left(1-\gamma_{\beta_{k, 0}, \rho}+\gamma_{\beta_{k, 0}, \rho} j a_{n}^{(\rho)}\right)\right.$. $\left.\prod_{\rho=0}^{P-1}\left[\left(1-\gamma_{\beta_{k, 0}, \rho}\right) \cos 2^{\rho} \varphi(t)+\gamma_{\beta_{k, 0}, \rho} \sin 2^{\rho} \varphi(t)\right]\right\}$, and the sum of the $M$ additive terms expanded from $\prod_{\rho=0}^{P-1}\left\{\cos 2^{\rho}\right.$ $\left.[h \pi-\varphi(t+i T)]-j a_{n-i}^{(\rho)} \sin 2^{\rho}[h \pi-\varphi(t+i T)]\right\}$ is expressed as $\sum_{\beta_{k, i}=0}^{M-1}\left\{\prod_{\rho=0}^{P-1}\left(1-\gamma_{\beta_{k, i}, \rho}-\gamma_{\beta_{k, i}, \rho} j a_{n-i}^{(\rho)}\right)\right.$ $\cdot \prod_{\rho=0}^{P-1}\left\{\left(1-\gamma_{\beta_{k, i}, \rho}\right) \cos 2^{\rho}[h \pi-\varphi(t+i T)]+\gamma_{\beta_{k, i}, \rho}\right.$ $\left.\left.\sin 2^{\rho}[h \pi-\varphi(t+i T)]\right\}\right\}$. Finally, the sum of the $M^{l}$ additive terms expanded from (22) becomes

$$
\begin{aligned}
& e^{j a_{n} \varphi(t)-j} \sum_{i=1}^{l-1} a_{n-i}[h \pi-\varphi(t+i T)] \\
&= \sum_{k=0}^{M^{l}-1}\left\{\prod_{\rho=0}^{P-1}\left(1-\gamma_{\beta_{k}, 0}+\gamma_{\beta_{k, 0}, \rho} j a_{n}^{(\rho)}\right)\right. \\
& \cdot \prod_{i=1}^{l-1} \prod_{\rho=0}^{P-1}\left(1-\gamma_{\beta_{k, i}, \rho}-\gamma_{\beta_{k, i}, \rho} j a_{n-i}^{(\rho)}\right) \\
& \cdot \prod_{\rho=0}^{P-1}\left[\left(1-\gamma_{\beta_{k, 0}, \rho}\right) \cos 2^{\rho} \varphi(t)+\gamma_{\beta_{k, 0}, \rho} \sin 2^{\rho} \varphi(t)\right] \\
& \cdot \prod_{i=1}^{l-1} \prod_{\rho=0}^{P-1}\left\{\left(1-\gamma_{\beta_{k, i}, \rho}\right) \cos 2^{\rho}[h \pi-\varphi(t+i T)]\right. \\
&\left.\left.+\gamma_{\beta_{k, i}, \rho} \sin 2^{\rho}[h \pi-\varphi(t+i T)]\right\}\right\}
\end{aligned}
$$

Note that since the first term in the expansion of $\prod_{\rho=0}^{P-1}\left[\cos 2^{\rho} \varphi(t)+j a_{n}^{(\rho)} \sin 2^{\rho} \varphi(t)\right]$ in (22) is $\left\{\prod_{\rho=0}^{P-1}\left(1-\gamma_{\beta_{k, 0}, \rho}+\gamma_{\beta_{k, 0}, \rho} j a_{n}^{(\rho)}\right) \quad \cdot \quad \prod_{\rho=0}^{P-1}\left[\left(1-\gamma_{\beta_{k, 0}, \rho}\right)\right.\right.$ $\left.\left.\cos 2^{\rho} \varphi(t)+\gamma_{\beta_{k, 0}, \rho} \sin 2^{\rho} \varphi(t)\right]\right\}_{\beta_{k, 0}=0}=\prod_{\rho=0}^{P-1} \cos 2^{\rho} \varphi(t)=$ $(\sin M \varphi(t)) /(M \sin \varphi(t))$, all the terms with $\beta_{k, 0}=0$ (total $M^{l-1}$ terms) in (25) will add up to $(\sin M \varphi(t))$ $/(M \sin \varphi(t)) e^{-j \sum_{i=1}^{l-1} a_{n-i}[h \pi-\varphi(t+i T)]}$, which is exactly the second term in the brackets on the RHS of (20), so that these terms will disappear in the decomposition of $h_{a_{n-l+1}, a_{n-l+2}, \ldots, a_{n}}(t)$. Therefore, $h_{a_{n-l+1}, a_{n-l+2}, \ldots, a_{n}}(t)$ comprises $M^{l-1}(M-1)$ terms from (20) and (25), i.e.,

$$
h_{a_{n-l+1}, a_{n-l+2}, \ldots, a_{n}}(t)=\sum_{\substack{k=1 \\ \beta_{k, 0} \neq 0}}^{M^{l}-1} \alpha_{k, n} h_{k}(t)
$$

where

$$
\begin{aligned}
\alpha_{k, n}= & \prod_{\rho=0}^{P-1}\left(1-\gamma_{\beta_{k, 0}, \rho}+\gamma_{\beta_{k, 0}, \rho} j a_{n}^{(\rho)}\right) \\
& \cdot \prod_{i=1}^{l-1} \prod_{\rho=0}^{P-1}\left(1-\gamma_{\beta_{k, i}, \rho}-\gamma_{\beta_{k, i}, \rho} j a_{n-i}^{(\rho)}\right)
\end{aligned}
$$


is a complex coefficient determined by index $k$ and data symbols $a_{n-i}, i=0,1, \ldots, l-1$, and

$$
\begin{aligned}
h_{k}(t)= & \bar{w}_{l}(t) \prod_{\rho=0}^{P-1}\left[\left(1-\gamma_{\beta_{k, 0}, \rho}\right)\right. \\
& \left.\cdot \cos 2^{\rho} \varphi(t)+\gamma_{\beta_{k, 0}, \rho} \sin 2^{\rho} \varphi(t)\right] \\
& \cdot \prod_{i=1}^{l-1} \prod_{\rho=0}^{P-1}\left\{\left(1-\gamma_{\beta_{k, i}, \rho}\right)\right. \\
& \cdot \cos 2^{\rho}[h \pi-\varphi(t+i T)] \\
& \left.+\gamma_{\beta_{k, i}, \rho} \sin 2^{\rho}[h \pi-\varphi(t+i T)]\right\}
\end{aligned}
$$

is a real-valued causal function, we call it the MMSE PAM pulse.

With $h_{a_{n-l+1}, a_{n-l+2}, \ldots, a_{n}}(t)$ being decomposed into MMSE PAM pulses, (18) is rewritten as

$$
\tilde{S}(t)=\sum_{n=-\infty}^{\infty} \sum_{\substack{k=1 \\ \beta_{k, 0} \neq 0}}^{M^{l}-1} a_{k, n} h_{k}(t-n T), \quad \text { for noninteger } h
$$

where

$$
a_{k, n}=a_{0, n-1} \alpha_{k, n}
$$

is the pseudosymbol associated with the $k$ th MMSE PAM pulse.

Similarly, from (19) we have

$$
\begin{aligned}
\tilde{S}(t) & =\sum_{n=-\infty}^{\infty} e^{j h \pi n} h_{0}(t-n T) \\
& +\sum_{n=-\infty}^{\infty} e^{j h \pi n} \sum_{\substack{k=1 \\
\beta_{k, 0} \neq 0}}^{M^{l}-1} \alpha_{k, n} h_{k}(t-n T), \text { for integer } h .
\end{aligned}
$$

We see that an $M$-ary CPM signal is approximated by a sum of $M^{l-1}(M-1)$ MMSE PAM components, plus a data-independent periodic component for the integer modulation index. By this MMSE PAM decomposition, CPM is now described as a combination of $M^{l-1}(M-1)$ linear time-invariant systems. The nonlinearity of phase modulation is embodied in the pseudosymbols $a_{k, n}$ or $\alpha_{k, n}$.

The first $M-1$ MMSE PAM pulses, numbered from $k=1$ to $M-1$, generally have infinite pulse duration (except for $h=q / M$ where $q$ is any integer). They constitute the first-order MMSE incremental pulse, and are therefore called the main MMSE PAM pulses. All other MMSE PAM pulses numbered from $k=M^{l-1}+1$ to $M^{l}-1$ are finite-duration pulses with duration $(L-l+1) T$ for $l=2,3, \ldots, L$.

From (27) and (30), we have $E\left[\alpha_{k, n}\right]=E\left[a_{k, n}\right]=0$, where $E[\cdot]$ denotes ensemble expectation over all transmitted data symbols, and

$$
E\left[\alpha_{k, n} \alpha_{i, m}^{*}\right]=E\left[a_{k, n} a_{i, m}^{*}\right]= \begin{cases}1, & m=n, i=k \\ 0, & \text { otherwise }\end{cases}
$$

Therefore, all the MMSE PAM components in an $M$-ary CPM signal are mutually independent. This is the fundamental advantage over the Laurent representation, by which the PAM components are independent only for those modulation indexes which are multiples of 0.5 .

The MMSE PAM pulses and associated pseudosymbols have explicit closed-form expressions for any modulation index and any symbol alphabet size, as can be seen in the above derivation. This motivates us to reformulate the Laurent representation to make the PAM decomposition more efficient, especially for a multilevel CPM signal (see Appendix B). More importantly, with the Laurent incremental pulses being defined, the reformulated Laurent representation also fits in the switched linear modulation models. Furthermore, under the reformulated Laurent representation, the modulation index $h$ of any $M$-ary $\left(M=2^{P}\right)$ CPM signal, or $2^{\rho} h, \rho=0,1, \ldots, P-1$ of any constitutional binary CPM signal, can be an integer.

\section{AutOCORRELATION AND APPROXIMATION ERROR EVALUATION}

First, let us derive the autocorrelation function of the approximated $M$-ary CPM signal $\tilde{S}(t)$, which is defined by

$$
R_{\tilde{S} \tilde{S}}(\tau)=\frac{1}{T} \int_{0}^{T} E\left[\tilde{S}^{*}(t) \tilde{S}(t+\tau)\right] d t .
$$

Using the switched linear modulation models and exploiting the properties of MMSE window function $\bar{w}_{l}(t)$, we can express $R_{\tilde{S} \tilde{S}}(\tau)$ in terms of $\bar{w}_{l}(t)$ and $\varphi(t)$, as shown in (33) at the bottom of the page, which is valid for both noninteger and integer modulation indexes. When $l=L$, the above autocorrelation function is the exact one of an $M$-ary CPM signal [4], [17].

It is of interest to know that from the above autocorrelation evaluation, $R_{\tilde{S} \tilde{S}}(\tau)$ is the same for all $1 \leq l \leq L$ when $|\tau| \geq$ $L T$. This implies that the approximation of an $M$-ary CPM signal, using either MMSE incremental pulses of order higher

$$
R_{\tilde{S} \tilde{S}}(\tau)=\left\{\begin{array}{c}
\frac{1}{T} \int_{0}^{(L-l+1) T} \bar{w}_{l}(t) \bar{w}_{l}(t+\tau) \prod_{i=-l+1}^{0} \frac{\sin M[\varphi(t+\tau-i T)-\varphi(t-i T)]}{M \sin [\varphi(t+\tau-i T)-\varphi(t-i T)]} d t \\
-\frac{1}{T} \int_{T}^{(L-l+1) T} \bar{w}_{l-1}(t) \bar{w}_{l-1}(t+\tau) \prod_{i=-l+2}^{0} \frac{\sin M[\varphi(t+\tau-i T)-\varphi(t-i T)]}{M \sin [\varphi(t+\tau-i T)-\varphi(t-i T)]} d t, \quad 0 \leq \tau \leq L T \\
\left(\frac{\sin M h \pi}{M \sin (h \pi)}\right)^{m-L} \frac{1}{T} \int_{0}^{T} \prod_{i=-L+1}^{0} \frac{\sin M[h \pi-\varphi(t-i T)]}{M \sin [h \pi-\varphi(t-i T)]} \prod_{i=-L+1}^{1} \frac{\sin M \varphi(t+\xi-i T)}{M \sin \varphi(t+\xi-i T)} d t, \\
\tau=m T+\xi, \quad m \geq L, \quad 0 \leq \xi \leq T \\
R_{\tilde{S} \tilde{S}}(-\tau), \quad \tau<0
\end{array}\right.
$$




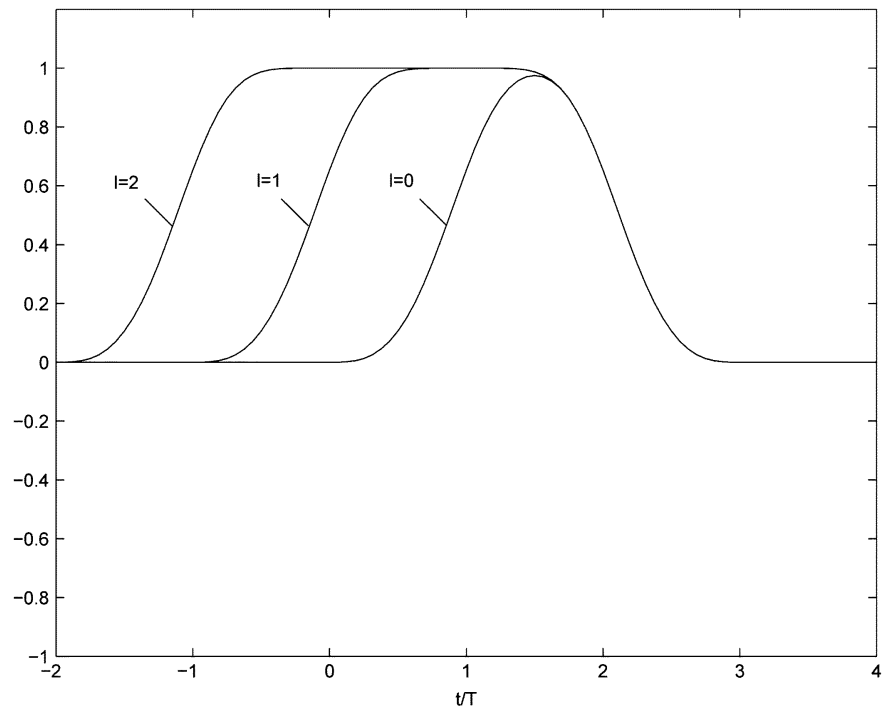

Fig. 2. MMSE window functions $\bar{w}_{l}(t)$ of quaternary $2 \mathrm{RC}$ scheme with $h=0.25$ for $l=0,1,2$.

than 1 or MMSE PAM pulses of number more than $M-1$, only improves the autocorrelation within $|\tau|<L T$.

Then, let us evaluate the approximation error when the $M$-ary CPM signal is represented by the superposition of linearly modulated pulses, which can be measured by

$$
\varepsilon^{2}=\frac{1}{T} \int_{0}^{T} E\left[|S(t)-\tilde{S}(t)|^{2}\right] d t .
$$

Since we can easily show, according to the switched linear modulation models, that

$$
E\left[S(t) \tilde{S}^{*}(t)\right]=E\left[|\tilde{S}(t)|^{2}\right]
$$

we have

$$
\begin{aligned}
& E\left[(S(t)-\tilde{S}(t)) \tilde{S}^{*}(t)\right] \\
& \quad=E\left[S(t) \tilde{S}^{*}(t)\right]-E\left[|\tilde{S}(t)|^{2}\right] \\
& \quad=0 \\
& E\left[|S(t)-\tilde{S}(t)|^{2}\right] \\
& \quad=1-E\left[|\tilde{S}(t)|^{2}\right]-E\left[(S(t)-\tilde{S}(t)) \tilde{S}^{*}(t)\right] \\
& \quad=1-E\left[|\tilde{S}(t)|^{2}\right] .
\end{aligned}
$$

Therefore, $\varepsilon^{2}$ is finally evaluated as

$$
\varepsilon^{2}=1-\frac{1}{T} \int_{0}^{T} E\left[|\tilde{S}(t)|^{2}\right] d t=1-R_{\tilde{S} \tilde{S}}(0) .
$$

Obviously, $\varepsilon^{2}$ will be zero when $l=L$.

Also note from (36) that the ensemble-averaged correlation between the error signal $S(t)-\tilde{S}(t)$ and the approximated signal $\tilde{S}(t)$ is always zero, which means that the error signal is orthogonal to the approximated signal, and hence, the approximation error is minimized for any given $l$.

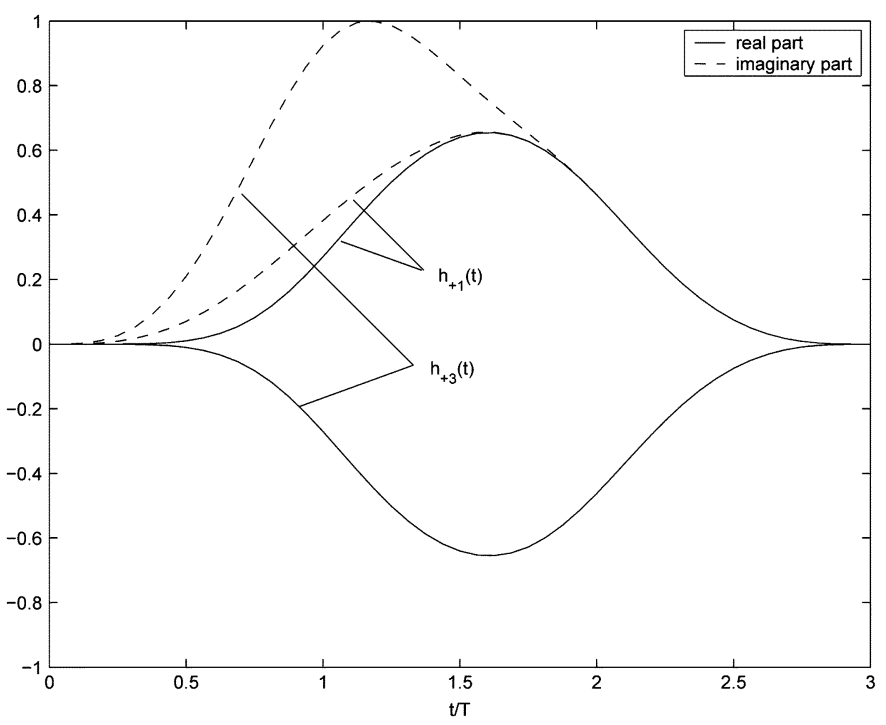

Fig. 3. First-order MMSE incremental pulses $h_{+3}(t)$ and $h_{+1}(t)$ of quaternary $2 \mathrm{RC}$ scheme with $h=0.25$.

\section{EXAMPLES}

The $M$-ary CPM signal with raised-cosine frequency pulse (i.e., the LRC scheme) is used to demonstrate the optimal signal modeling by the switched linear modulation models. Fig. 2 shows the MMSE window functions $\bar{w}_{l}(t)$ of a quaternary 2RC scheme (i.e., $M=4$ and $L=2$ ) with $h=0.25$ for $l=0,1,2$. The first-order MMSE incremental pulses $h_{+3}(t)$ and $h_{+1}(t)$ are shown in Fig. $3\left(h_{-1}(t)\right.$ and $h_{-3}(t)$ are not displayed because of the symmetry property). The second-order MMSE incremental pulses are shown in Fig. 4(a) and (b). Only 8 out of 16 complex-valued pulses are displayed due to the symmetry property. Fig. 5 shows the MMSE PAM pulses, where the three main MMSE PAM pulses are displayed in Fig. 5(a), and the rest of the nine MMSE PAM pulses are shown in Fig. 5(b) with extended scales. Table I summarizes how these MMSE PAM pulses are calculated according to (28). We also draw the nine MMSE PAM pulses shown in Fig. 5(b) using the same scales as those used in Fig. 5(a) [see the enclosed area on the left-hand side bottom of Fig. 5(a)] and find out that these pulses only carry a small amount of signal power, as compared with the main MMSE PAM pulses. Therefore, in most practical cases, we can use the main MMSE PAM pulses (or the first-order MMSE incremental pulses) to approximate the CPM signal without causing significant modeling error.

Fig. 6 shows the autocorrelation functions of the quaternary 2RC schemes with $h=0.25,0.5,1$ for $l=1,2$. Since $L=2$, the second-order autocorrelation functions are the exact autocorrelation functions of the quaternary $2 \mathrm{RC}$ schemes. The firstorder autocorrelation functions for $h=0.25,0.5$ are almost the same as their exact ones, whereas the first-order autocorrelation function for $h=1$ is slightly different from its exact version, with $R_{\tilde{S} \tilde{S}}(0)=0.967$. This means that the approximation errors using the first-order MMSE incremental pulses or the main MMSE PAM pulses for $h=0.25,0.5$ are negligible, whereas only $3.3 \%$ of signal power is ignored by signal approximation for $h=1$. Fig. 7 shows the approximation errors as functions of $h$ for $M$-ary LRC schemes with different $M$ and 


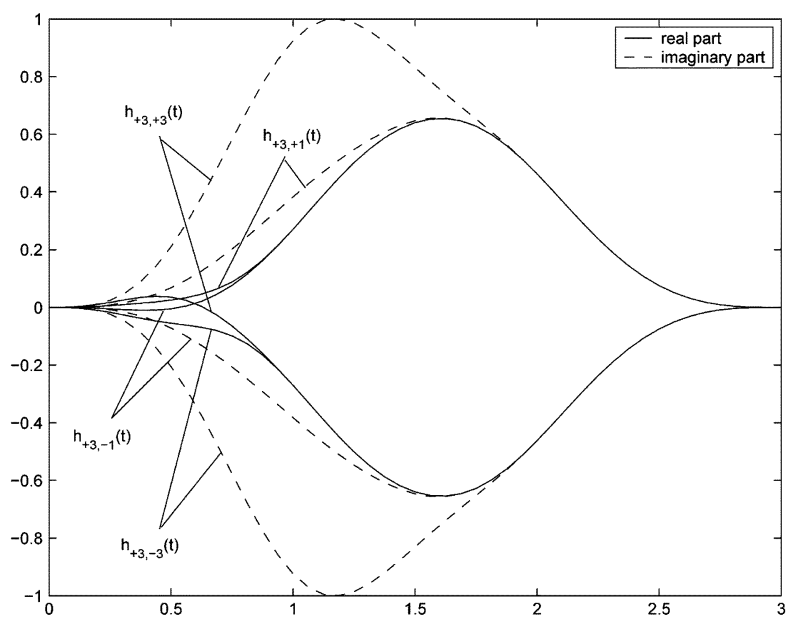

(a)

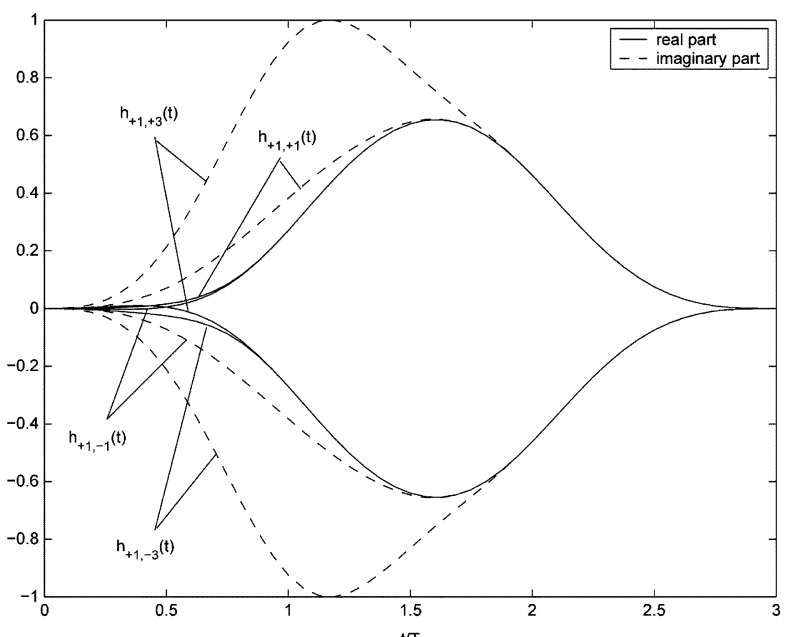

(b)

Fig. 4. Second-order MMSE incremental pulses of quaternary 2 RC scheme with $h=0.25$. (a) $h_{+3,+3}(t), h_{+3,+1}(t), h_{+3,-1}(t)$, and $h_{+3,-3}(t)$. (b) $h_{+1,+3}(t), h_{+1,+1}(t), h_{+1,-1}(t)$, and $h_{+1,-3}(t)$.

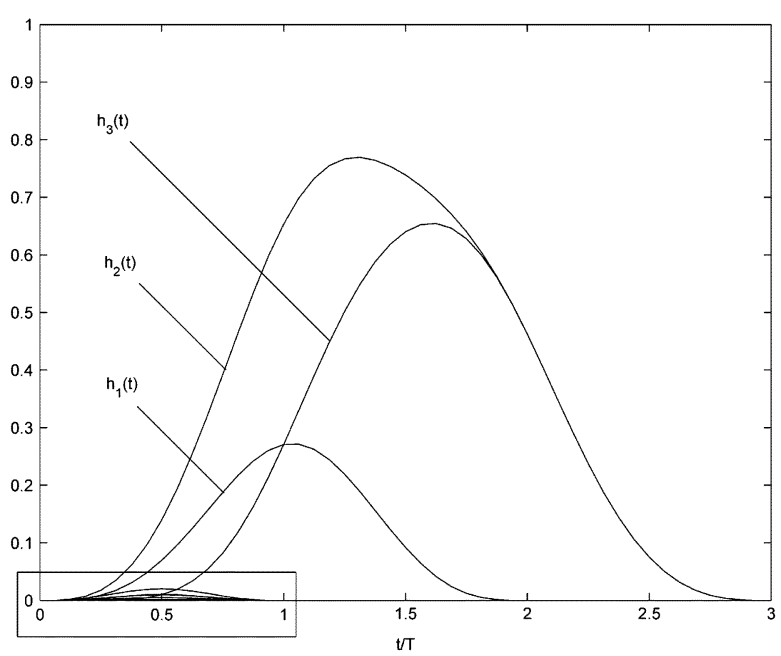

(a)

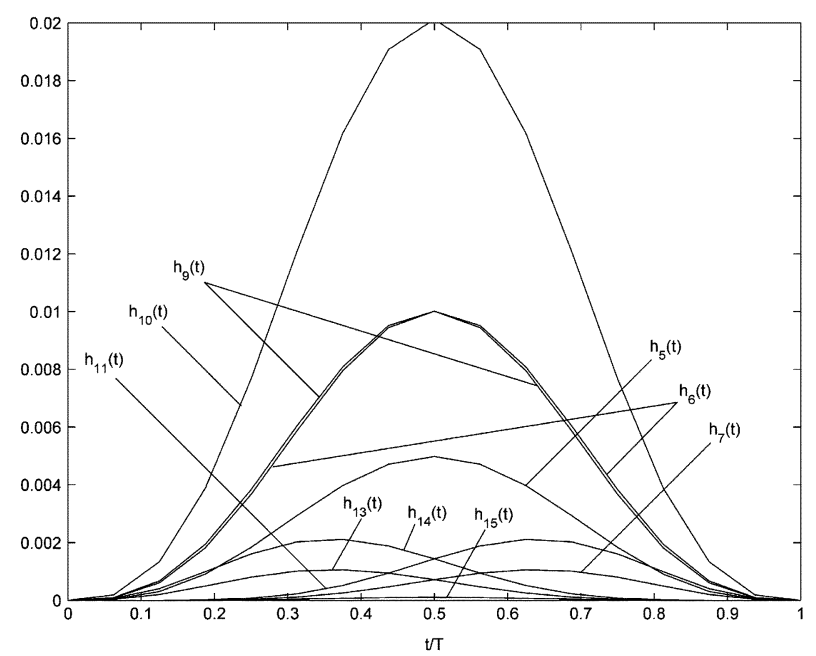

(b)

Fig. 5. MMSE PAM pulses of quaternary 2RC scheme with $h=0.25$. (a) Main MMSE PAM pulses. (b) Other MMSE PAM pulses.

$L$ values, using the first- and second-order MMSE incremental pulses (or the corresponding numbers of MMSE PAM pulses), respectively. We see that the approximation errors generally increase as $M, L$, or $h$ increase for a given order of approximation. However, by increasing the order of approximation, we can meet any approximation-error requirement for any $M, L$, and $h$ values. For most practical binary and quaternary CPM signals, the first-order approximation is already sufficient to achieve efficient signal modeling with satisfactory approximation precision. For example, the first-order approximation error for the quaternary $2 \mathrm{RC}$ scheme with $h=0.25$ can be found to be $2 \times 10^{-4}$ from Fig. 7(a), which means that only $0.02 \%$ of signal power is ignored by this signal approximation.

Finally, let us compare the MMSE-optimal approximation with the Laurent approximation approach. Since the Laurent incremental pulses have been defined in Appendix B, this comparison can be easily performed under the same switched linear modulation modes. The approximation errors as a function of $h$ for $M$-ary LRC schemes with different $M$ and $L$ values, using the first-order Laurent incremental pulses and the first-order MMSE incremental pulses, respectively, are shown in Fig. 8. We see that the $\varepsilon^{2} \sim h$ curves using Laurent incremental pulses are discontinuous functions of $h$, with discontinuity points located at $h=(2 m) /(M)$, where $m$ are positive integers (the Laurent representation is valid at these $h$, but invalid in the close vicinities of these $h$ ), whereas the $\varepsilon^{2} \sim h$ curves using MMSE incremental pulses are continuous functions of $h$, and the approximation errors are always smaller (or equal at some $h$ values). Once the parameters $M$ and $L$ are given and an approximation-error threshold is specified, these $\varepsilon^{2} \sim h$ curves also provide us with indications as to where the valid regions of $h$ are. For example, if we want to model the quaternary $(M=4) 2 \mathrm{RC}(L=2) \mathrm{CPM}$ signal with modeling error less than 0.1 using the Laurent approximation, the valid modulation index, according to Fig. 8(a), will be $0<h<0.44, h=0.5,0.57<h<0.81$, and $h=1$. If we want to model the CPM signal with the same $M$ and $L$ parameters and the same modeling-error threshold, but using the MMSE-optimal approximation, any $h$ will be valid. 
TABLE I

CALCUlation of MMSE PAM PUlses For QuATERnARy 2RC SChEME $(M=4$ AND $L=2)$

\begin{tabular}{|c|c|c|c|c|c|c|c|}
\hline \multirow{5}{*}{$k$} & \multicolumn{2}{|c|}{$\beta_{k, i}$} & \multicolumn{4}{|c|}{$\gamma_{\beta_{k, i}, \rho}$} & \multirow{5}{*}{$h_{k}(t)$} \\
\hline & \multirow{2}{*}{\multicolumn{2}{|c|}{$i$}} & \multicolumn{4}{|c|}{$i$} & \\
\hline & & & \multicolumn{2}{|c|}{1} & \multicolumn{2}{|c|}{0} & \\
\hline & \multirow[t]{2}{*}{1} & \multirow[t]{2}{*}{0} & \multicolumn{4}{|c|}{$\rho$} & \\
\hline & & & 1 & 0 & 1 & 0 & \\
\hline 0 & 0 & 0 & 0 & 0 & 0 & 0 & \\
\hline 1 & 0 & 1 & 0 & 0 & 0 & 1 & $\bar{w}_{1}(t) \sin \varphi(t) \cos 2 \varphi(t)$ \\
\hline 2 & 0 & 2 & 0 & 0 & 1 & 0 & $\bar{w}_{1}(t) \cos \varphi(t) \sin 2 \varphi(t)$ \\
\hline 3 & 0 & 3 & 0 & 0 & 1 & 1 & $\bar{w}_{1}(t) \sin \varphi(t) \sin 2 \varphi(t)$ \\
\hline 4 & 1 & 0 & 0 & 1 & 0 & 0 & \\
\hline 5 & 1 & 1 & 0 & 1 & 0 & 1 & $\bar{w}_{2}(t) \sin \varphi(t) \cos 2 \varphi(t) \sin [h \pi-\varphi(t+T)] \cos 2[h \pi-\varphi(t+T)]$ \\
\hline 6 & 1 & 2 & 0 & 1 & 1 & 0 & $\bar{w}_{2}(t) \cos \varphi(t) \sin 2 \varphi(t) \sin [h \pi-\varphi(t+T)] \cos 2[h \pi-\varphi(t+T)]$ \\
\hline 7 & 1 & 3 & 0 & 1 & 1 & 1 & $\bar{w}_{2}(t) \sin \varphi(t) \sin 2 \varphi(t) \sin [h \pi-\varphi(t+T)] \cos 2[h \pi-\varphi(t+T)]$ \\
\hline 8 & 2 & 0 & 1 & 0 & 0 & 0 & \\
\hline 9 & 2 & 1 & 1 & 0 & 0 & 1 & $\bar{w}_{2}(t) \sin \varphi(t) \cos 2 \varphi(t) \cos [h \pi-\varphi(t+T)] \sin 2[h \pi-\varphi(t+T)]$ \\
\hline 10 & 2 & 2 & 1 & 0 & 1 & 0 & $\bar{w}_{2}(t) \cos \varphi(t) \sin 2 \varphi(t) \cos [h \pi-\varphi(t+T)] \sin 2[h \pi-\varphi(t+T)]$ \\
\hline 11 & 2 & 3 & 1 & 0 & 1 & 1 & $\bar{w}_{2}(t) \sin \varphi(t) \sin 2 \varphi(t) \cos [h \pi-\varphi(t+T)] \sin 2[h \pi-\varphi(t+T)]$ \\
\hline 12 & 3 & 0 & 1 & 1 & 0 & 0 & \\
\hline 13 & 3 & 1 & 1 & 1 & 0 & 1 & $\bar{w}_{2}(t) \sin \varphi(t) \cos 2 \varphi(t) \sin [h \pi-\varphi(t+T)] \sin 2[h \pi-\varphi(t+T)]$ \\
\hline 14 & 3 & 2 & 1 & 1 & 1 & 0 & $\bar{w}_{2}(t) \cos \varphi(t) \sin 2 \varphi(t) \sin [h \pi-\varphi(t+T)] \sin 2[h \pi-\varphi(t+T)]$ \\
\hline 15 & 3 & 3 & 1 & 1 & 1 & 1 & $\bar{w}_{2}(t) \sin \varphi(t) \sin 2 \varphi(t) \sin [h \pi-\varphi(t+T)] \sin 2[h \pi-\varphi(t+T)]$ \\
\hline
\end{tabular}

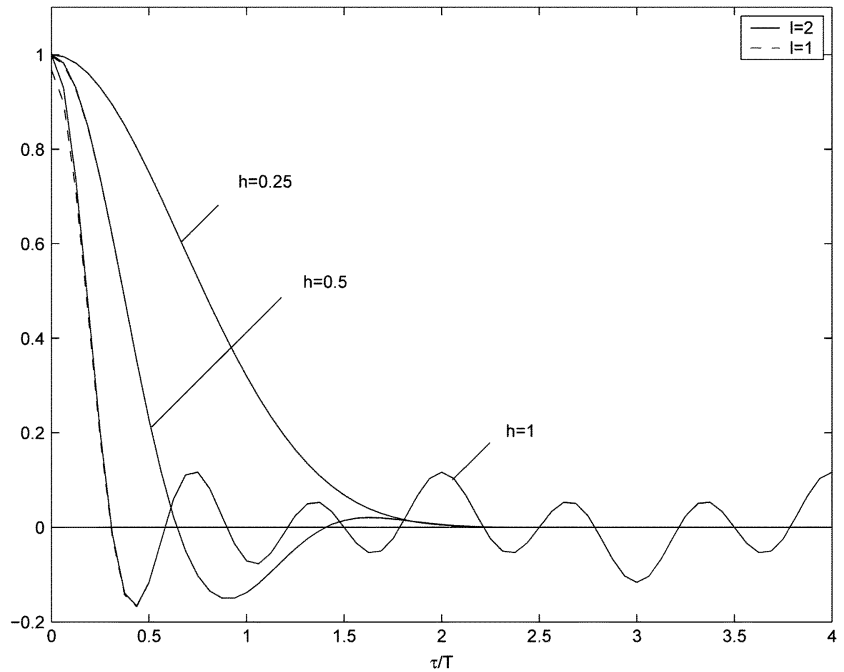

Fig. 6. Autocorrelation functions of quaternary $2 \mathrm{RC}$ with $h=0.25,0.5,1$ for $l=1,2$.

\section{CONCLUSION}

We have shown that any $M$-ary CPM signal with any modulation index can be exactly expressed as the superposition of a finite number of linearly modulated pulses. These linearly modulated pulses can be either the MMSE incremental pulses, by which the CPM signal can be represented by the switched linear modulation models, or the MMSE PAM pulses. Explicit closedform formulas for these linearly modulated pulses and associated pseudosymbols are also developed. The proposed novel CPM signal linear modulation representation will simplify the CPM signal analysis, since all the linearly modulated signal components are mutually independent. When using lower order MMSE incremental pulses or a small number of MMSE PAM pulses to approximate the CPM signal, the approximation error is minimized in the MMSE sense, resulting in the MMSE-optimal CPM signal approximation, superior to the Laurent approximation approach.

\section{APPENDIX A}

\section{LAURENT SigNAL COMPONENT FOR CPM SIGNAL}

This appendix introduces the notion of a Laurent signal component for an $M$-ary CPM signal. It is shown that the Laurent signal component can be expressed as a Laurent complex pulse 


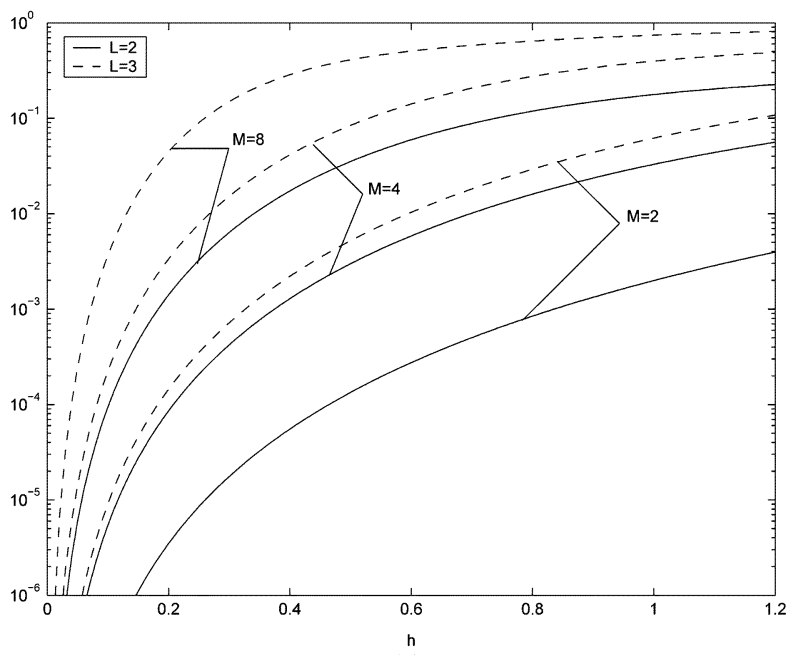

(a)

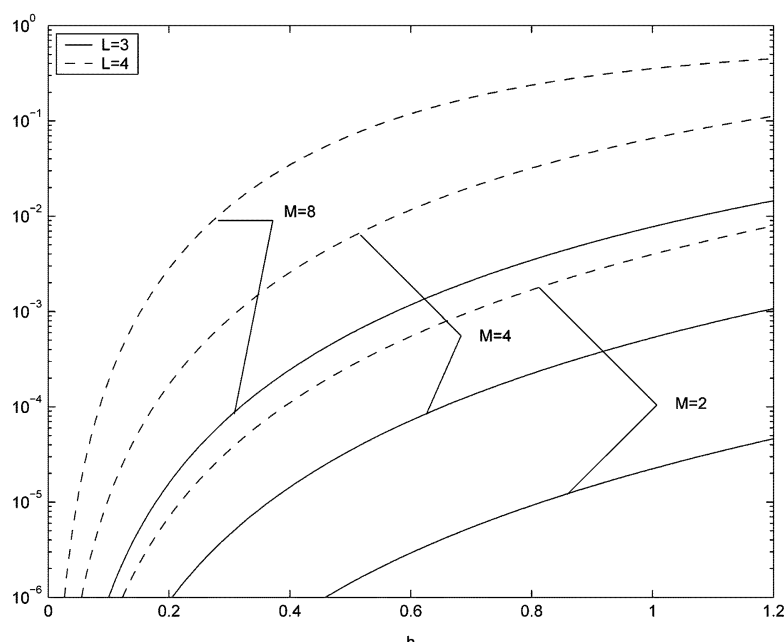

(b)

Fig. 7. Approximation errors versus modulation index for $M$-ary LRC schemes with $M=2,4,8$ and $L=2,3,4$ using (a) the first-order MMSE incremental pulses, and (b) the second-order MMSE incremental pulses.

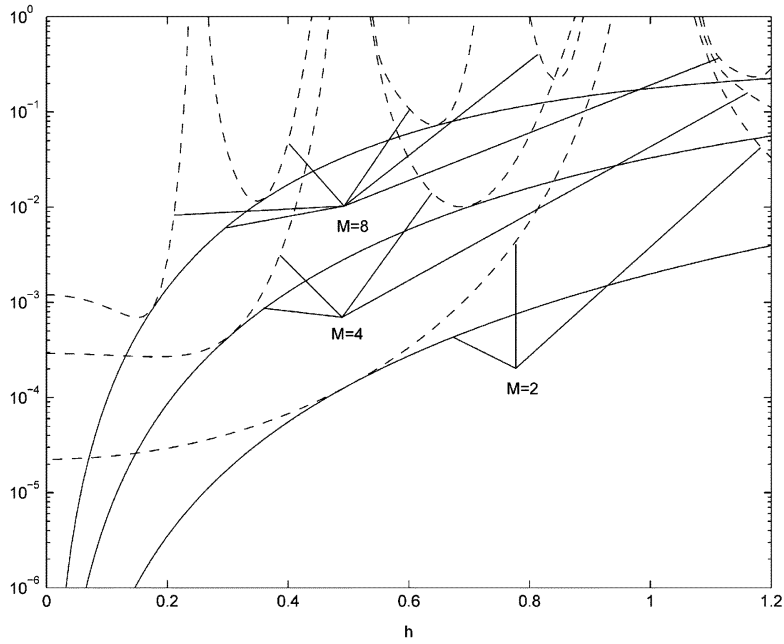

(a)

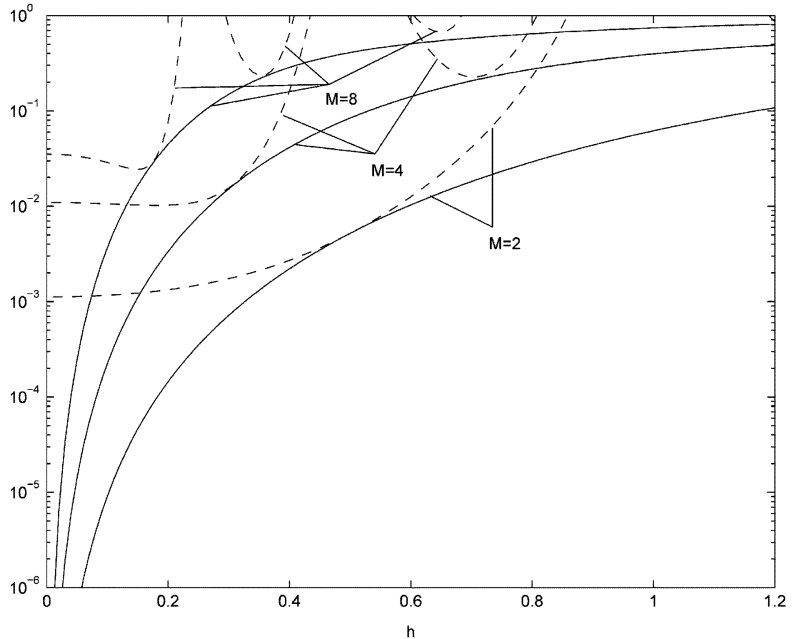

(b)

Fig. 8. Approximation errors versus modulation index using the first-order Laurent incremental pulses (dashed lines) and the first-order MMSE incremental pulses (solid lines), respectively, for (a) $M$-ary LRC schemes with $M=2,4,8$ and $L=2$, and (b) $M$-ary LRC schemes with $M=2,4,8$ and $L=3$.

multiplied by a phase state symbol, where the Laurent complex pulse is a windowed complex exponential.

Let us begin with a binary CPM signal (i.e., $M=2$ and $a_{n}= \pm 1$ ). From [5] and [7], $S(t)$ in (1) can be decomposed into a sum of $2^{L-1}$ PAM components for noninteger $h$, i.e., $S(t)=\sum_{n=-\infty}^{\infty} \sum_{k=0}^{2^{L-1}-1} a_{k, n} h_{k}(t-n T)$, or, a sum of one data-independent periodic component and $2^{L-1}$ data-dependent PAM components for integer $h$, i.e., $S(t)=\sum_{n=-\infty}^{\infty} e^{j h \pi n} h_{0}(t-n T)+$ $\sum_{n=-\infty}^{\infty} e^{j h \pi n} \sum_{k=1}^{2^{L-1}} \alpha_{k, n} h_{k}(t-n T)$. Since $a_{0, N}=$ $a_{0, N-1} e^{j h \pi a_{N}}$ from (3) for noninteger $h$, we see that a symbol $a_{N}$ at time $t=N T$ is encoded in the transition from $a_{0, N-1}$ to $a_{0, N}$, and the contribution of $a_{N}$ to the transmitted signal waveform is represented by the PAM component $a_{0, N} h_{0}(t-N T)$. Considering the impact of all previously transmitted date symbols, which is included in the PAM component $a_{0, N-1} h_{0}(t-N T+T)$, we define $S\left(t, a_{N}\right)=a_{0, N-1} h_{0}(t-N T+T)+a_{0, N} h_{0}(t-N T)$ as the main Laurent signal component associated with symbol $a_{N}$. For integer $h$, since $\alpha_{1, N}=j a_{N}$ from [7], we know that the contribution of $a_{N}$ to the transmitted signal waveform is represented by the PAM component $e^{j h \pi N} \alpha_{1, N} h_{1}(t-N T)$. Therefore, including the data-independent periodic component, the main Laurent signal component is defined as $S\left(t, a_{N}\right)=\sum_{i=-\infty}^{\infty} e^{j h \pi i} h_{0}(t-i T)+e^{j h \pi N} \alpha_{1, N} h_{1}(t-$ $N T)$. Using the Laurent representation formulas derived from [5] and [7], $S\left(t, a_{N}\right)$ can be finally expressed as $S\left(t, a_{N}\right)=a_{0, N-1} S_{a_{N}}(t-N T)$ for noninteger $h$, or $S\left(t, a_{N}\right)=e^{j h \pi N} S_{a_{N}}(t-N T)$ for integer $h$, where

$$
S_{a_{n}}(t)=w(t) e^{j a_{n} \varphi(t)}
$$

is a windowed complex exponential with real-valued window function

$$
w(t)=\left\{\begin{array}{l}
\prod_{i=-L}^{-1} \frac{\sin \varphi(t-i T)}{\sin (h \pi)} \prod_{i=1}^{L} \frac{\sin [h \pi-\varphi(t-i T)]}{\sin (h \pi)}, \\
\prod_{\substack{i=-\infty \\
i \neq 0}}^{\infty} \cos \varphi(t-i T), \quad \text { for noninteger } h
\end{array}\right.
$$


and phase-shift function $a_{n} \varphi(t)$. We call $S_{a_{n}}(t)$ the main Laurent complex pulse associated with symbol $a_{n}$.

The concepts of the main Laurent signal component and main Laurent complex pulse can be easily extended to $M$-ary CPM signals, by defining the main Laurent signal component of the $M$-ary CPM signal as the product of its $P$ constitutional binary CPM signals' main Laurent signal components. It is found that the main Laurent signal component can be always expressed as a complex-valued pulse, i.e., the main Laurent complex pulse of the $M$-ary CPM signal, multiplied by a phase state symbol. The main Laurent complex pulse also has the same expression as (A1). However, the window function now becomes $w(t)=$ $\prod_{\rho=0}^{P-1} w^{(\rho)}(t)$, a product of the $P$ binary CPM signals' window functions, where each $w^{(\rho)}(t)$ is calculated using (A2), with the phase shift function replaced by $2^{\rho} \varphi(t)$ and the modulation index by $2^{\rho} h$. Note that for some noninteger $h=q / 2^{v}, v=$ $1,2, \ldots, P-1$ and $q$ is any odd number, the modulation index $2^{\rho} h$ may become an integer when $\rho \geq v$. Also note that for noninteger $2^{\rho} h$, we have $\prod_{i=-\infty}^{-L-1}\left(\sin 2^{\rho} \varphi(t-i T)\right) /\left(\sin 2^{\rho} h \pi\right)=$ $\prod_{i=L+1}^{\infty}\left(\sin 2^{\rho}[h \pi-\varphi(t-i T)]\right) /\left(\sin 2^{\rho} h \pi\right)=1$ for $-T \leq$ $t \leq(L+1) T$, due to the property of $\varphi(t)$ (2). Therefore, the window function for any $M$-ary CPM signal can be generally expressed, in terms of $\varphi(t)$, as

$$
\begin{aligned}
w(t)= & \prod_{i=-\infty}^{-1} \prod_{\rho=0}^{v-1} \frac{\sin 2^{\rho} \varphi(t-i T)}{\sin 2^{\rho} h \pi} \prod_{\rho=v}^{P-1} \cos 2^{\rho} \varphi(t-i T) \\
& \cdot \prod_{i=1}^{\infty} \prod_{\rho=0}^{v-1} \frac{\sin 2^{\rho}[h \pi-\varphi(t-i T)]}{\sin 2^{\rho} h \pi} \prod_{\rho=v}^{P-1} \cos 2^{\rho} \varphi(t-i T) .
\end{aligned}
$$

For noninteger $h$, with which none of $2^{\rho} h, \rho=0,1, \ldots, P-1$ is an integer, we simply replace $v$ with $P$ and ignore the term $\prod_{\rho=v}^{P-1} \cos 2^{\rho} \varphi(t-i T)$ in (A3). Equation (A3) also applies to integer $h$ by assuming $v=0$ and ignoring the terms $\prod_{\rho=0}^{v-1}\left(\sin 2^{\rho} \varphi(t-i T)\right) /\left(\sin 2^{\rho} h \pi\right)$ and $\prod_{\rho=0}^{v-1}\left(\sin 2^{\rho}[h \pi-\varphi(t-i T)]\right) /\left(\sin 2^{\rho} h \pi\right)$, since all $2^{\rho} h, \rho=0,1, \ldots, P-1$ will be integers.

Extending the main Laurent signal component and main Laurent complex pulse concepts to include $l$ consecutively transmitted data symbols, we define the Laurent signal component associated with data symbols $a_{N-l+1}, a_{N-l+2}, \ldots, a_{N}$ for any $M$-ary CPM signal as the sum of all PAM pulses modulated by their respective pseudosymbols, which are only related to these $l$ data symbols, as well as the phase state symbol $a_{0, N-l}$ (for noninteger $h$ ) or $e^{j h \pi N}$ (for integer $h$ ), including the data-independent periodic component for integer $h$, and find out that it can be always expressed as

$$
\begin{aligned}
& S\left(t, a_{N-l+1}, a_{N-l+2}, \ldots, a_{N}\right) \\
& \quad=a_{0, N-l} S_{a_{N-l+1}, a_{N-l+2}, \ldots, a_{N}}(t-N T), \text { for noninteger } h
\end{aligned}
$$

$$
\begin{aligned}
& S\left(t, a_{N-l+1}, a_{N-l+2}, \ldots, a_{N}\right) \\
& \quad=e^{j h \pi N} S_{a_{N-l+1}, a_{N-l+2}, \ldots, a_{N}}(t-N T), \text { for integer } h .
\end{aligned}
$$

The Laurent complex pulse $S_{a_{N-l+1}, a_{N-l+2}, \ldots, a_{N}}(t)$ associated with the $l$ data symbols $a_{N-l+1}, a_{N-l+2}, \ldots, a_{N}$ is also derived as a windowed complex exponential with window function $w_{l}(t)$ and phase shift function $\sum_{i=-l+1}^{0} a_{N+i} \varphi(t-i T)$, i.e.,

$$
S_{a_{N-l+1}, a_{N-l+2}, \ldots, a_{N}}(t)=w_{l}(t) \cdot e^{j \sum_{i=-l+1}^{0} a_{N+i} \varphi(t-i T)}
$$

where the window function is found to be

$$
\begin{aligned}
w_{l}(t)= & \prod_{i=-\infty}^{-l} \prod_{\rho=0}^{v-1} \frac{\sin 2^{\rho} \varphi(t-i T)}{\sin 2^{\rho} h \pi} \prod_{\rho=v}^{P-1} \cos 2^{\rho} \varphi(t-i T) \\
& \cdot \prod_{i=1}^{\infty} \prod_{\rho=0}^{v-1} \frac{\sin 2^{\rho}[h \pi-\varphi(t-i T)]}{\sin 2^{\rho} h \pi} \prod_{\rho=v}^{P-1} \cos 2^{\rho} \varphi(t-i T)
\end{aligned}
$$

which is a finite-duration pulse for noninteger $h$ with $w_{l}(t)=0$ for $t \leq-l T$ and $t \geq(L+1) T$, or an infinite-duration pulse for integer $h$.

Some interesting properties of $w_{l}(t)$ can be also identified as follows:

$$
\begin{aligned}
& \begin{aligned}
w_{l}(t) & \prod_{\rho=0}^{v-1} \frac{\sin 2^{\rho} \varphi(t+l T-T)}{\sin 2^{\rho} h \pi} \prod_{\rho=v}^{p-1} \cos 2^{\rho} \varphi(t+l T-T) \\
& =w_{l-1}(t)
\end{aligned} \\
& w_{l}(t) \prod_{\rho=0}^{v-1} \frac{\sin 2^{\rho}[h \pi-\varphi(t)]}{\sin 2^{\rho} h \pi} \prod_{\rho=v}^{P-1} \cos 2^{\rho} \varphi(t)=w_{l-1}(t+T)
\end{aligned}
$$

$w_{l}(t)=w_{l-1}(t), \quad$ for $t \geq(L-l+1) T$.

For integer $h, w_{0}(t)$ is a periodic function and has the same alternative expression as (11).

\section{APPENDIX B \\ REFORMULATION OF LAURENT REPRESENTATION}

Based on the Laurent signal component expression derived in Appendix A, and following the same procedure as described in Section III, we can express an $M$-ary CPM signal exactly as the superposition of the $L$ th-order Laurent incremental pulse $h_{a_{n-L+1}, a_{n-L+2}, \ldots, a_{n}}(t)$ plus a data-independent periodic component for integer $h$, similar to (16) and (17). The $l$ th-order Laurent incremental pulse is found to be

$$
\begin{gathered}
h_{a_{n-l+1}, a_{n-l+2}, \ldots, a_{n}}(t)=w_{l}(t) \cdot e^{-j \sum_{i=-l+1}^{-1} a_{n+i}[h \pi-\varphi(t-i T)]} \\
\left.\cdot\left(e^{j a_{n} \varphi(t)}-\prod_{\rho=0}^{v-1} \frac{\sin 2^{\rho[h \pi-\varphi(t)]}}{\sin 2^{\rho} h \pi} \prod_{\rho=v}^{P-1} \cos 2^{\rho} \varphi(t)\right) \cdot \quad \text { (B } 1\right)
\end{gathered}
$$

Thus an $M$-ary CPM signal can be approximated by the same switched linear modulation models, but with Laurent incremental pulses.

Furthermore, following the same procedure as described in Section IV, but using equation $e^{j a_{n}^{(\rho)} 2^{\rho} \phi(t)}=\left(\sin 2^{\rho}[h \pi-\right.$ $\phi(t)]) /\left(\sin 2^{\rho} h \pi\right)+e^{j 2^{\rho} h \pi a_{n}^{(\rho)}}\left(\sin 2^{\rho} \phi(t)\right) /\left(\sin 2^{\rho} h \pi\right)$ for noninteger $2^{\rho} h$, the $l$ th-order Laurent incremental pulse defined 
by (B1) can be decomposed as a sum of $M^{l-1}(M-1)$ Laurent PAM pulses.

The $l$ th-order approximation of the $M$-ary CPM signal using Laurent PAM pulses has the same expressions as (29) and (31), but $\alpha_{k, n}$ and $h_{k}(t)$ are now evaluated, respectively, by

$$
\begin{aligned}
\alpha_{k, n}= & \prod_{\rho=0}^{v-1} e^{j \gamma_{\beta_{k, 0}, \rho} 2^{\rho} h \pi a_{n}^{(\rho)}} \prod_{\rho=v}^{P-1}\left(1-\gamma_{\beta_{k}, 0}+\gamma_{\beta_{k, 0}, \rho} j a_{n}^{(\rho)}\right) \\
& \cdot \prod_{i=1 \rho=0}^{l-1 v-1} e^{-j \gamma_{\beta_{k, i}, \rho} 2^{\rho} h \pi a_{n-i}^{(\rho)}} \prod_{\rho=v}^{P-1}\left(1-\gamma_{\beta_{k, i}, \rho}-\gamma_{\beta_{k, i}, \rho} j a_{n-i}^{(\rho)}\right)
\end{aligned}
$$

$$
\begin{aligned}
h_{k}(t)= & w_{l}(t) \prod_{\rho=0}^{v-1}\left[\left(1-\gamma_{\beta_{k, 0}, \rho}\right) \frac{\sin 2^{\rho}[h \pi-\varphi(t)]}{\sin 2^{\rho} h \pi}\right. \\
& \left.+\gamma_{\beta_{k, 0}, \rho} \frac{\sin 2^{\rho} \varphi(t)}{\sin 2^{\rho} h \pi}\right] \prod_{\rho=v}^{P-1}\left[\left(1-\gamma_{\beta_{k, 0}, \rho}\right) \cos 2^{\rho} \varphi(t)\right. \\
& \left.+\gamma_{\beta_{k, 0}, \rho} \sin 2^{\rho} \varphi(t)\right] \\
& \cdot \prod_{i=1}^{l-1} \prod_{\rho=0}^{v-1}\left[\left(1-\gamma_{\beta_{k, i}, \rho}\right) \frac{\sin 2^{\rho} \varphi(t+i T)}{\sin 2^{\rho} h \pi}\right. \\
& \left.+\gamma_{\beta_{k, i}, \rho} \frac{\sin 2^{\rho}[h \pi-\varphi(t+i T)]}{\sin 2^{\rho} h \pi}\right] \\
& \cdot \prod_{\rho=1}^{P-1}\left\{\left(1-\gamma_{\beta_{k, i}, \rho}\right) \cos 2^{\rho}[h \pi-\varphi(t+i T)]\right. \\
& \left.+\gamma_{\beta_{k, i}, \rho} \sin 2^{\rho}[h \pi-\varphi(t+i T)]\right\}
\end{aligned}
$$

\section{REFERENCES}

[1] T. Aulin and C.-E. W. Sundberg, "Continuous phase modulation-Part I: Full response signaling," IEEE Trans. Commun., vol. COM-29, no. 3, pp. 196-209, Mar. 1981.

[2] T. Aulin, N. Rydbeck, and C.-E. W. Sundberg, "Continuous phase modulation-Part II: Partial response signaling," IEEE Trans. Commun., vol. COM-29, no. 3, pp. 210-225, Mar. 1981.

[3] J. B. Anderson, T. Aulin, and C.-E. W. Sundberg, Digital Phase Modulation. New York: Plenum, 1986.

[4] J. G. Proakis, Digital Communications, 3rd ed. New York: McGrawHill, 1995.

[5] P. A. Laurent, "Exact and approximate construction of digital phase modulation by superposition of amplitude modulated pulses (AMP)," IEEE Trans. Commun., vol. COM-34, no. 2, pp. 150-160, Feb. 1986.

[6] U. Mengali and M. Morelli, "Decomposition of $M$-ary CPM signals into PAM waveforms," IEEE Trans. Inf. Theory, vol. 41, no. 5, pp. 1265-1275, Sep. 1995.

[7] X. Huang and Y. Li, "The PAM decomposition of CPM signal with integer modulation index," IEEE Trans. Commun., vol. 51, no. 4, pp. 543-546, Apr. 2003.

[8] G. K. Kaleh, "Simple coherent receivers for partial response continuous phase modulation," IEEE J. Sel. Areas Commun., vol. 7, no. 9, pp. 1427-1436, Dec. 1989.
[9] G. Colavolpe and R. Raheli, "Reduced-complexity detection and phase synchronization of CPM signals," IEEE Trans. Commun., vol. 45, no. 9 , pp. 1070-1079, Sep. 1997.

[10] - "Noncoherent sequence detection of continuous phase modulations," IEEE Trans. Commun., vol. 47, no. 9, pp. 1303-1307, Sep. 1999.

[11] A. N. D'Andrea, A. Ginesi, and U. Mengali, "Frequency detectors for CPM signals," IEEE Trans. Commun., vol. 43, no. 2-4, pp. 1828-1837, Feb.-Apr. 1995.

[12] A. Napolitano and C. M. Spooner, "Cyclic spectral analysis of continuous-phase modulated signals," IEEE Trans. Signal Process., vol. 49, no. 1, pp. 30-44, Jan. 2001.

[13] B. E. Rimoldi, "A decomposition approach to CPM," IEEE Trans. Inf. Theory, vol. 34, no. 2, pp. 260-270, Mar. 1988

[14] X. Huang and Y. Li, "Simple CPM receivers based on a switched linear modulation model," IEEE Trans. Commun., vol. 53, no. 7, pp. 1100-1103, Jul. 2005.

[15] J. Huber and W. Liu, "An alternative approach to reduced-complexity CPM-receivers," IEEE J. Sel. Areas Commun., vol. 7, no. 9, pp. 1437-1449, Dec. 1989.

[16] P. Moqvist and T. Aulin, "Orthogonalization by principal components applied to CPM," IEEE Trans. Commun., vol. 51, no. 11, pp. 1838-1845, Nov. 2003.

[17] T. Aulin and C.-E. W. Sundberg, "An easy way to calculate power spectra for digital FM," IEE Proc. Commun., Radar, Signal Process. pt. F, vol. 130, no. 6, pp. 519-526, Oct. 1983.

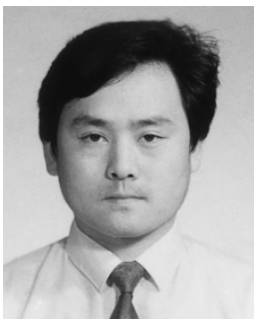

Xiaojing Huang (M'99) was born in Hubei, China, on October 3, 1963. He received the Bachelor, Master, and Ph.D. degrees from Shanghai Jiao Tong University, Shanghai, China, in 1983, 1986, and 1989 , respectively, all in electronic engineering.

From 1989 to 1994, he worked in the Electronic Engineering Department of Shanghai Jiao Tong University, where he had been an Associate Professor since 1991. From 1994 to 1997, he was the Chief Engineer with Shanghai Yang Tian Science and Technology Company Ltd., Shanghai, China. In 1998, he joined Motorola Australian Research Center, Botany, NSW, Australia, where he had been a Senior Research Engineer since 1999 and a Principal Research Engineer since 2003. He is currently an Associate Professor in the School of Electrical, Computer and Telecommunications Engineering, University of Wollongong, Wollongong, NSW, Australia. His research interests are in communications theory, digital signal processing, and wireless communication networks.

Dr. Huang is currently a voting member of the IEEE 802.15 Working Group, and has been actively involved in the IEEE 802.15.3a alternative ultra-wideband physical layer standard.

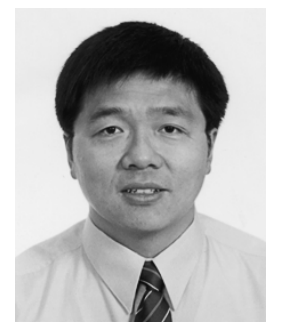

Yunxin Li (S'92-M'95-SM'01) was born in Sichuan Province, China, in 1963. In 1983, he received the Bachelor degree from Chongqing University, Chongqing, China, and the Master and Ph.D. degrees from the University of New South Wales, Sydney, Australia, and the University of Sydney, Sydney, Australia, respectively, in 1990 and 1995.

He served in various senior technical and management positions in the China Ministry of Railway, Shanghai University, University of Sydney, Motorola, and Freescale. He is currently the Director of the Twincall Education Centre, Sydney, Australia. His current research interest is in communications and networking technologies and their applications in innovative education system. 